\title{
Análisis sinóptico de un evento extremo y persistente de lluvia sobre el estado de Río Grande do Sul, Brasil, en mayo de 2004
}

\author{
Synoptic analysis of an extreme and persistent rainfall event over state of Rio \\ Grande do Sul, Brazil, in May 2004
}

\author{
Raidel Báez Prieto e Mateus da Silva Teixeira \\ Universidade Federal de Pelotas, Brasil \\ raidelbp@gmail.com; mateus.teixeira@ufpel.edu.br
}

\begin{abstract}
Resumen
Entre el 4 y 9 de mayo de 2004, en parte del estado de Rio Grande do Sul (RS), Brasil, se han registrado precipitaciones superiores a $300 \mathrm{~mm}$. En la región este de este estado, principalmente en la costa, se ha producido la mayor acumulación de lluvia. Un análisis sinóptico de este período demuestra una vaguada persistente al oeste de RS en los medios y altos niveles de la tropósfera, en los tres primeros días de este evento. Esta vaguada inicia un vórtice ciclónico en los niveles medios de la troposfera para los siguientes días. El movimiento ascendente asociado a esta vaguada se quedó semi-estacionario sobre el estado de RS y actuó en casi la totalidad de los niveles de la troposfera durante gran parte de este período. Además, se observó en los niveles troposféricos, altos valores de humedad relativa desde la superficie hasta 300 hPa, sobre todo el estado de RS, mayor al 70\%. El día 9 de mayo de 2004, el vórtice ciclónico ha perdido su configuración y se ha distanciado de RS. El estado de RS queda dominado por una circulación anticiclónica y por niveles troposféricos más secos, lo que desfavorece la ocurrencia precipitaciones en esta región. Durante todo el periodo analizado, se pudo observar que la configuración en la troposfera media se amplió hasta los niveles más altos, pero no hasta la superficie. Además, se observó una circulación ciclónica en 850 hPa sobre el estado de RS, pero ninguna ciclogénesis se produjo en la superficie. Una ciclogénesis en superficie se observó sobre la costa del estado de Sao Paulo (SP), el 5 de mayo. Este ciclón se traslado al sur, cerca a las costas de los estados de RS e Santa Catarina (SC), el 08 de mayo. Una comparación del comportamiento atmosférico con la precipitación registrada en el estado de RS entre 3 y 9 puede sugerir que este centro de baja presión no tiene influencia directa en la precipitación acumulada observada en este período. Además, el 8 y 9 de mayo, este centro de baja presión se debilitó y se alejó de la costa de los estados de RS y SC. Por lo tanto, a pesar de los débiles y bajos niveles de advección de temperatura y de convergencia del flujo de humedad observados sobre esta región, la vaguada en la media y baja troposfera persistente, lo que favorece al persistente movimiento ascendente, que junto a la profunda humedad por días, parece ser el principal responsable de las grandes cantidades de precipitaciones registradas en el estado de RS.
\end{abstract}

Palabras-claves: lluvias extremas, lluvias persistentes.

\section{Abstract}

Between 04 to 09 May 2004, part of the state of Rio Grande do Sul (RS), Brazil; have recorded rainfall above 300mm. The east region of this state, mainly on the coast, had the highest rainfall accumulation occurred. A synoptic analysis of this period has shown a persistent trough west of RS in high and middle levels of troposphere, in the first three days of this event. This trough started a cyclonic vortice in the mid-lowtropospheric levels in the following days. The upward movement associated with this trough stayed semi-stationary over the state of RS and acted in almost entire tropospheric layer during great part of this period. In addition, it was observed that this tropospheric layer had high values of relative humidity from surface up to $300 \mathrm{hPa}$, over the state of RS - greater than $70 \%$. In 09 May 2004, the cyclonic vortice has lost their configuration and has distanced from RS. The state of RS was dominated by an anticyclonic circulation and by a drier tropospheric layer, what disfavors rainfall occurrence in this region. During all analyzed period, it could be observed that the configuration in the middle troposphere has extended up to higher levels, but the same does not occurred to the surface. Also, a cyclonic circulation in $850 \mathrm{hPa}$ was observed over the state of RS, but any cyclogenesis has occurred at surface. A surface cyclogenesis was observed over the coast of the state of Sao Paulo (SP), in 05 May. This cyclone has moved to south, approaching the coast of the states of RS e Santa Catarina (SC), in 08 May. A comparison of the atmospheric behavior with the rainfall recorded in the state of RS between 03 and 09 May suggest that this low-pressure center has no direct influence in the cumulative rainfall observed in this period. In addition, in 08 and 09 May, this low-pressure center weakened and move away from the coast of the states of RS and SC. Therefore, although the weak low-level temperature advection and lowlevel moisture flux convergence observed over this region, a persistent mid-low-tropospheric trough, what favor persistent upward movement, together with a deep and very humid layer, seems to be the main responsible to the great amounts of rainfall registered in the state of RS. 


\section{Introducción}

La variabilidad y el cambio climático están presentes como factores de presión en las decisiones de la civilización actual y futura. Prever condiciones que pueden dar lugar a desastres ambientales extremas es uno de los retos científicos de hoy en día (Andrade, 2005; CLIMANÁLISE 2006). Existe una necesidad en la predicción de eventos de precipitación extrema, su inicio y mantenimiento por días (NUNES; DA SILVA, 2013; TEIXEIRA; Satyamurty, 2010). Brasil ha estado sufriendo con varios desastres ambientales, causados principalmente por la lluvia intensa, lluvia prolongada, intensos vientos (Grimm, 2009). El Estado de Rio Grande do Sul (RS), en el sur de Brasil, con un fuerte papel en la economía, depende del comportamiento de las lluvias. Eventos de fuertes lluvias se producen de forma homogénea a lo largo del año en esta región de Brasil (BARBIERI, 2005; BRITTO et al, 2006). Los desastres relacionados con las lluvias extremas y persistentes a menudo se producen de momento, siendo importante conocer que inicia y mantiene estos fenómenos, para poder crear un sistema de alerta que mitigue y reduzca sus impactos, brindando una mejor información a la población.

\section{Objetivo}

Hacer el análisis sinóptico del entorno que fue favorable para desarrollar y mantener un evento extremo de lluvia persistente, ocurrido en RS, Brasil, en mayo de 2004.

\section{Material y métodos}

Para el estudio fueron utilizados datos diarios de precipitación, disponibles en el Instituto Nacional de Meteorología - INMET por medio del Banco de Datos Meteorológicos, para 10 años (2004-2013) de observación para RS, tomando 18 estaciones meteorológicas bien distribuidas geográficamente por todo el estado como se muestra en la figura 1.

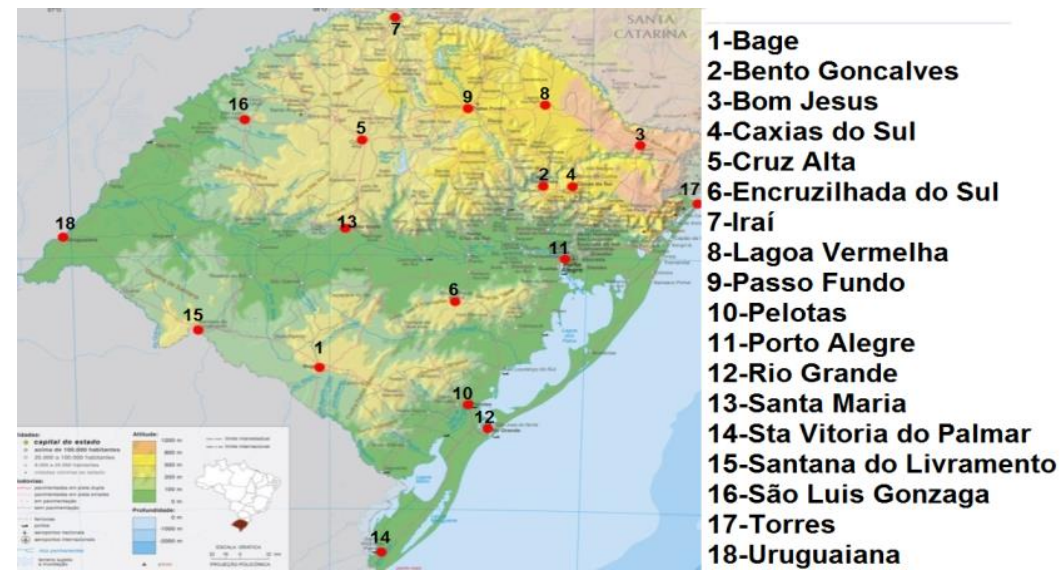

Figura 1- Representación de las 18 estaciones en Rio Grande do Sul con datos de precipitación (2004-2013) que fueron utilizados en el estudio.

Fueron usadas tres características da la precipitación para definir un evento extremo de lluvia persistente, (i) duración, (ii) acumulado en el período y (iii) cobertura espacial, las mismas se obtuvieron por medio de los cuantiles. Un evento extremo de lluvia persistente es todo período de lluvia que ocasionó un gran acumulado de precipitación, en un gran período de tiempo con gran efecto en la región.

Para obtener la primera característica de la lluvia, en cada una das 18 estaciones meteorológicas, fueron contabilizados los días con lluvia superior a $1 \mathrm{~mm}$, siendo organizados y agrupados los datos en períodos con días 
consecutivos de lluvia y sus acumulados. Se selecciona el caso que supere el cuantil 0.9 o $90 \%$ de la persistencia en días dentro de los períodos antes agrupados y alcance el mayor acumulado de lluvia en ( $\mathrm{mm})$. Para cumplir la segunda característica de la lluvia, el caso seleccionado debe superar el $50 \%$ da media estacional $(\mathrm{mm})$ dentro de los 10 años de estudio. La tercera y última característica de lluvia por la cual se filtra el caso, fue a cobertura espacial que representa, al mantenerse en más de 5 estaciones meteorológicas, que representan más del $30 \%$ de las 18 total que se tienen en RS. Obteniendo finalmente durante os 10 años de estudio el caso de lluvia persistente extrema, con gran cobertura, intensidad e duración en la región. A partir de lo antes expuesto, se propone hacer un estudio sinóptico del caso seleccionado, con datos de reanálisis II del National Centers for Environmental Prediction (NCEP). Estos datos contienen informaciones de temperatura, humedad, viento, omega, altura geopotencial y presión en diferentes niveles atmosféricos, con espacio entre rejillas de $2,5^{\circ}$ de latitud por $2,5^{\circ}$ de longitud.

\section{Resultados}

Una vez que se realizan las 3 etapas de filtraje que se explican en la metodología, es seleccionado el caso más extremo y persistente de lluvia. Se representa el caso en la Figura 1, donde se puede observar el comportamiento en las 5 estaciones meteorológicas donde afectó, con acumulado mayor de 300 (mm) y con persistencia de 6 días continuos de lluvia (4-9 mayo de 2004). El mismo, actúa con mayor intensidad principalmente en la región costera al este de RS, como en la ciudad de Torres.

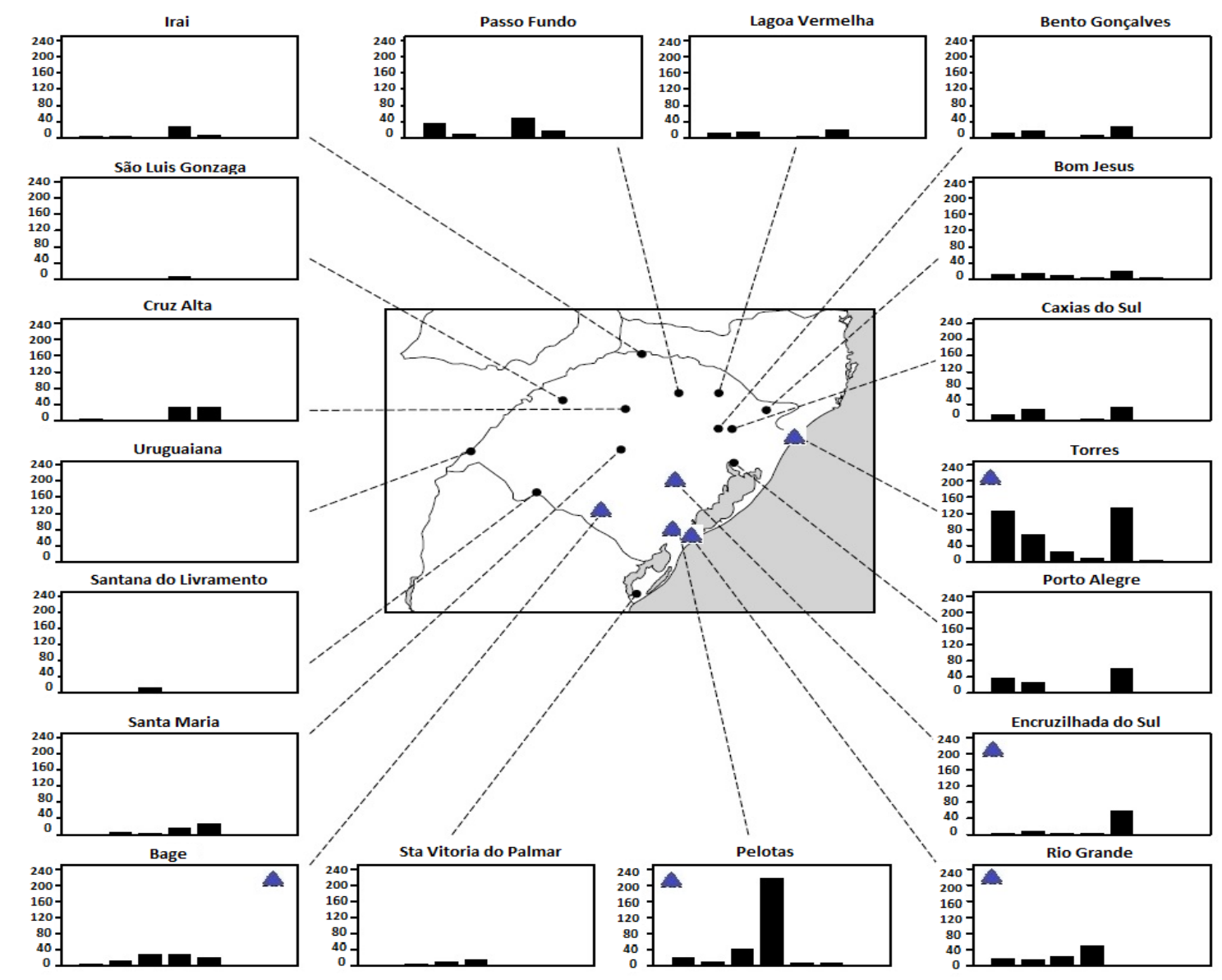

Figura 1- Comportamiento de las lluvias en el caso de estudio. En cada estación meteorológica se representa un gráfico con ordenada que se refiere a la lluvia acumulada en milímetros y abscisa que se refiere a su duración en días. 
La Figura 2 muestra los valores de alta humedad desde los primeros días en los niveles de la atmósfera. Como muestra la figura 3-A, para el primer día del evento se tiene en altos niveles divergencia positiva del viento, favoreciendo a la convergencia y a las corrientes ascendentes desde la superficie. Las figuras 4$\mathrm{AB}, 5-\mathrm{AB}$ e 6- $\mathrm{AB}$, muestran una vaguada persistente al oeste de RS en los altos y medios niveles de la troposfera, que forma un vórtice ciclónico en altos niveles (baja fría) que se profundiza hasta los bajos niveles de la troposfera durante los siguientes días.

La Figura 4-C muestra una circulación cerrada en superficie para los últimos días del evento, en la costa del Estado de São Paulo (SP) el día 5 de mayo. Este ciclón se trasladó al sur y se acercó a las costas de los estados de RS y Santa Catarina (SC) para el día 8. Una comparación del comportamiento atmosférico con la precipitación registrada en el Estado entre el 4 y 9 de mayo sugiere que este centro de baja presión, no tiene ninguna influencia directa de las precipitaciones observadas durante este período.

Para los días 8 y 9 de mayo, este centro de baja presión se aleja de las costas de los Estados de RS y SC, la vaguada persiste durante días, figuras 5 y 6, manteniendo en casi todos los niveles gran humedad, Figura 2, que parece ser la principal responsable de las continuas precipitaciones registradas en el Estado de RS. Desde la superficie hasta la troposfera superior, $300 \mathrm{hPa}$, se observan altos valores de humedad relativa, mayor al 70\%, Figura 2 .
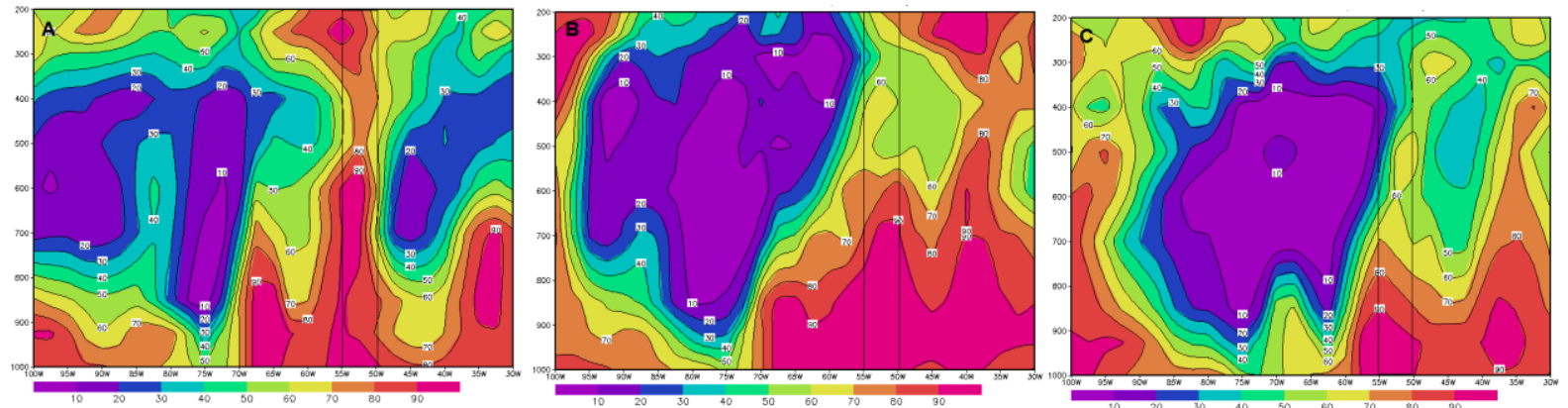

Figura 2- Representación de la humedad promedio a partir de un corte en vertical entre los $-34^{\circ}$ y $-27^{\circ}$ latitud sur, en todos los niveles de la atmósfera (longitud de RS está dentro del rectángulo). A- Día antes del comienzo de la lluvia, B- Día dentro del caso, C- Día final del caso.

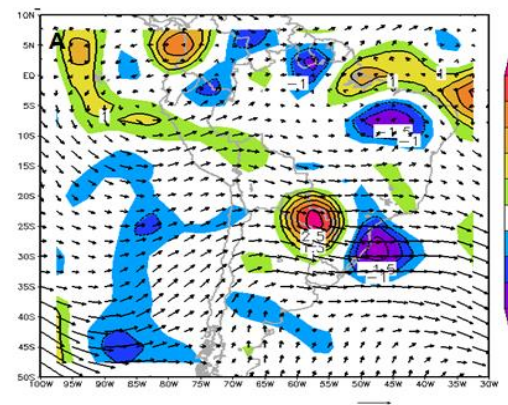

70
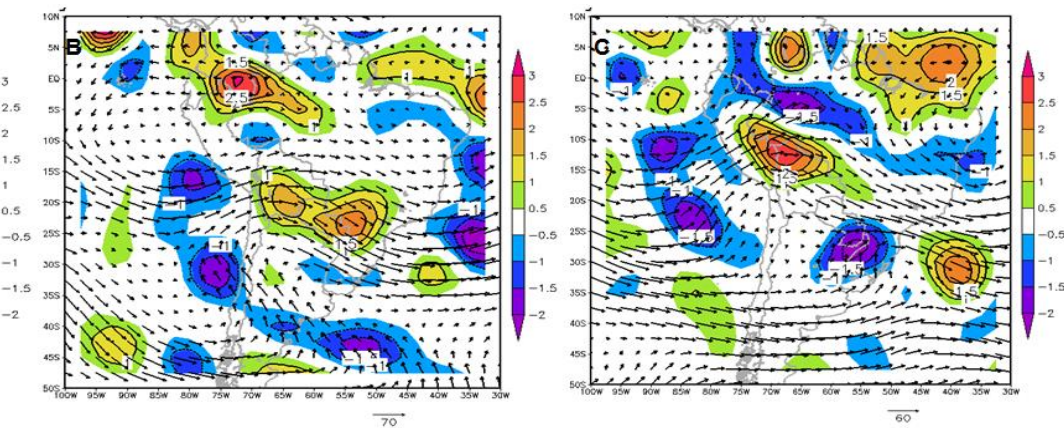

Figure 3- Representación de la divergencia y magnitudes del viento $200 \mathrm{hPa}$

A- Día antes del comienzo de la lluvia, B- Día dentro del caso, C- Día final del caso.

Para el último día del caso, 9 de mayo, el vórtice ciclónico perdió su configuración distanciándose de RS. La región está dominada en altos niveles, $200 \mathrm{hPa}$, por la divergencia negativa del viento, provocando la circulación anticiclónica en la superficie, que en unión a una masa de aire seco y sin humedad relativa significativa, no favorecen la ocurrencia de lluvias en esta región como se muestra en las figuras 2-C, 3-C, 4-C, 5-C, 6-C. 

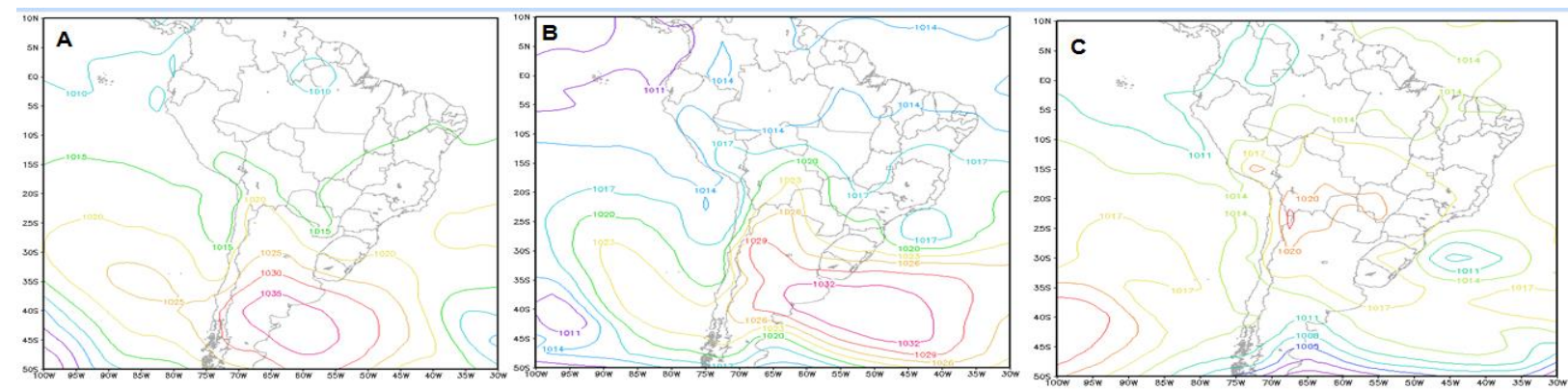

Figura 4 - Representación de la presión del nivel medio del mar (MSLP)

A- Día antes del comienzo de la lluvia, B- Día dentro del caso, C- Día final del caso.
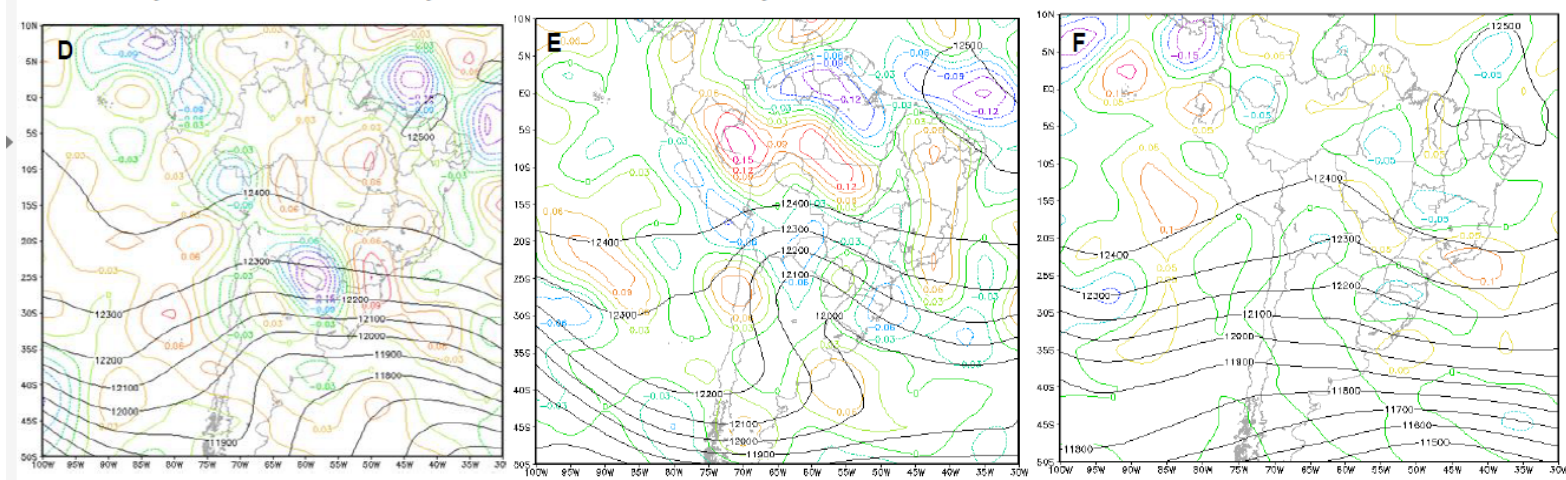

Figure 5- Representación de la Altura Geopotencial y Omega (200 hPa)

A- Día antes del comienzo de la lluvia, B- Día dentro del caso, C- Día final del caso.
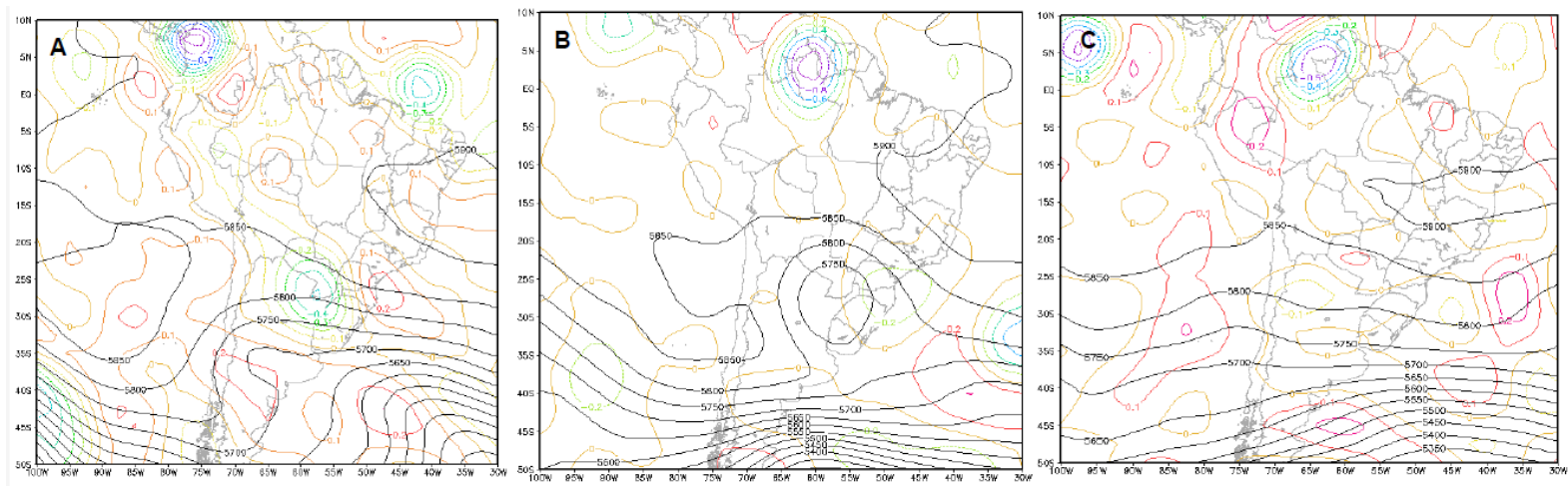

Figure 6- Representación de la Altura Geopotencial y Omega (500 hPa)

A- Día antes del comienzo de la lluvia, B- Día dentro del caso, C- Día final del caso.

\section{Conclusiones}

Según las referencias previas a este trabajo, la distribución de ocurrencia de eventos de lluvia extrema es durante todo el año sobre RS, pero el caso de lluvia persistente con el mayor acumulado estudiado se produce en el otoño.

Este caso analizado en este trabajo, indica cómo el patrón de circulación sinóptica un vórtice ciclónico de altos niveles (baja fría), donde la humedad y los movimientos ascendentes (omega), mantuvieron la persistencia de la lluvia. Este fue un ejemplo de un cambio de estación de año (otoño), con las condiciones tanto tropicales como extratropicales, causando la inestabilidad que junto a la influencia del vórtice ciclónico de altos niveles (baja fría), mantienen las condiciones para la lluvia continua con poco movimiento en la región, acabando su desarrollo cuando ambas condiciones atmosféricas finalizan. 


\section{Agradecimientos}

Agradecimientos a revisores, colaboradores y Bolsa del Conselho Nacional de Desenvolvimento Científico e Tecnológico CNPq.

\section{Referencias}

ANDRADE, K. M. Climatologia e comportamento dos sistemas frontais sobre a América do Sul. 2005. 185f. Dissertação (Mestrado en Meteorología) - Instituto Nacional de Pesquisas Espaciais-INPE, São José dos Campos.

BARBIERI, P. R. B. Caracterização da estação chuvosa nas regiões sul e sudeste do Brasil associado con a circulação. 2005. 118f. Dissertação (Mestrado en Meteorología) Instituto Nacional de Pesquisas EspaciaisINPE., São José dos Campos.

BRITTO, F.; BARLETTA, R.; MENDOÇA M. Regionalização Sazonal e mensal da precipitación pluvial máxima no estado do Rio Grande do Sul. Revista Brasileira de Climatología, ISSN 1980-0055X, Associação Brasileira de Climatología, Presidente Prudente, SP. v. 2, n 2, p. 35-51, 2006.

CLIMANÁLISE, Boletim de Monitoramento e Análise Climática, Cachoeira Paulista, SP, INPE/CPTEC, v 21, n.1, p. 11-24, 2006.

GRIMM, A. M. Clima da Região Sul do Brasil. In: CAVALCANTI, I. F. A.; FERREIRA, N. J.; JUSTI DA SILVA, M. G. A.; SILVA DIAS, M. A. F. Tempo e clima no Brasil. SP.: Ed. Oficina de Textos, 2009. p. 259-274.

NUNES, A. B.; DA SILVA, G. C. Climatology of extreme rainfall events in eastern and northern Santa Catarina state: present and future climate. Revista Brasileira de Geofísica (Impresso), v.31, p.1-13, 2013.

TEIXEIRA, M. S.; SATYAMURTY, P. Dynamical and synoptic characteristics of heavy rainfall episodes in southern Brazil. Monthly Weather Review, v.135, p.598-617, 2007. 